\title{
The practice of climate simulation and its social and political context
}

\section{A.C. Petersen}

Netherlands Environmental Assessment Agency, P.0. Box 303, 3720 AH Bilthoven, the Netherlands. Email: arthur.petersen@pbl.nl

Manuscript received: July 2008; accepted: September 2008

\begin{abstract}
The practice of climate simulation takes place in a polarized social and political context. In this paper some methodological aspects of the practice of climate simulation are addressed and the potential value-ladenness of modelling assumptions is discussed. I claim that there is clearly a plurality of values guiding climate simulation efforts with climate scientists themselves also commonly holding different political views on the climate-change problem. There exist climate models of varying levels of concreteness and with different basic assumptions, and the modelling approaches behind these models are valued differently by different groups of climate scientists. The social and political context in which the climate modelling is done plays a role in these value judgements. In order to prevent one particular group of models from dominating the field for social and/or political reasons, the climate-modelling community should acknowledge the vital and necessary role of plurality in the practice of climate science and should stimulate reflection within this practice. Finally, while the IPCC partly addresses the issue by presenting model ensembles, the uncertainties in climate simulation should be better communicated to policy makers and politicians.
\end{abstract}

Keywords: climate change, computer simulation, scientific practice, social and political values, uncertainty, value-ladenness

\section{Introduction}

The subject of climate change is imbued with scientific dissensus as to precisely what is happening, and will happen, with the climate. Part of this dissensus is related to the large uncertainties associated with climate simulation (see e.g., Petersen, 2006b, Ch. 6). Furthermore, there is disagreement on the appropriate political response vis-à-vis anthropogenic climate change (e.g., to what extent do we want to limit anthropogenic interference with the climate system? What should we do to mitigate the likely causes of climate change? To what extent should we prepare to adapt to it?). Perceptions of the climate-change risk vary widely both across the globe and within societies. Thus, the uncertainties are large - with climate simulation being a significant contributor to these uncertainties - and the stakes are high. This puts the problem of anthropogenic climate change in the category of policy problems that are in need of a 'post normal' problem-solving strategy (Funtowicz \& Ravetz, 1993; Petersen, 2006a; Van der Sluijs et al., 2008; Swart et al., 2008).
This paper addresses the influence of polarized social and political perspectives on the practice of climate simulation, particularly focusing on simulations that are used for global climate change detection and attribution studies and for global average projections. Climate simulations clearly play an important role in climate science. These simulations involve mathematical models that are implemented on computers and that numerically describe processes in the climate system. Like the history of numerical weather prediction, the history of climate science is strongly related to the history of the computer. There are two main reasons why simulation is so important in climate science. First, computers removed a barrier in meteorological practice: the speed with which calculations could be done has been enhanced tremendously. It is simply impractical to do the calculations required for climate simulations without the use of computers. Secondly, numerically simulations are an important tool for climate scientists because controlled real experiments with the climate as a whole are impossible. If we want to 'experimentally' manipulate climate to assess the impact of varying input 
parameters, we need to perform such manipulations on a representation of the climate system. That is, we must carry out computer simulations of theoretical climate models and vary the input parameters.

In the following sections, first the social and political dimensions of the climate-change debate are introduced. Subsequently, some methodological aspects of the practice of climate simulation are addressed prior to discussing the potential value-ladenness of modelling assumptions. It is argued that there is a plurality of judgmental values guiding climate simulation efforts. Climate scientists themselves hold different political views on the climate-change problem and this may to some extent affect their results and conclusions. This paper concludes with a discussion on the importance of maintaining a plurality of climate-simulation models.

\section{Social and political dimension of the climate-change debate}

In the climate-change debate, the stakes are indeed high. From the perspective of international relations and political science, the negotiating positions of countries within the United Nations Framework Convention on Climate Change (UNFCCC) are largely based on how they politically perceive the climate change issue, e.g. as an issue that is treated equitably, that is providing a country with opportunities, and/or that - if not enough is done - is negatively affecting the country (Van Asselt et al., 2008).

Some key players in the economy feel their existence threatened by calls for drastic reductions of $\mathrm{CO}_{2}$ emissions. By 2030 the macro-economic costs for multi-gas mitigation, consistent with emission trajectories towards stabilization between 445 and $710 \mathrm{ppm} \mathrm{CO}$-eq, are estimated at between a $3 \%$ decrease of global Gross Domestic Product (GDP) and a small increase in GDP, compared to the baseline figure (IPCC, 2007c, p. 11). Over the period $2007-2030$ this amounts to a maximum reduction of the GDP growth rate by 0.13 percentage points/ year (that is, a small but still significant fraction of the projected average yearly GDP growth rates of $2-3 \%$ ). Note that some regions and sectors (obviously those involved in the oil and coal industry) will bear a particularly large share of these economic costs, while some other sectors, such as agriculture outside of the tropics, will - at least initially and with some adaptation - benefit from climate change. The stakes are also high for those who, through the projected climate change, risk damage to themselves or to things they value. For instance, some ecosystems are projected to become irreversibly damaged, species will become extinct, some developing small-island states risk disappearance with continued sea-level rise, food production may suffer in many areas, et cetera (see IPCC, 2007b). And finally, the stakes are high for those players who see business opportunities for more environmentally friendly technology.
Future climate policies will most likely be a mixture of adaptation by societies to human-induced climate change and mitigation of the cause(s) of this change, mainly by reducing the emissions of greenhouse gasses. We are in the tragic situation that as a consequence of increased greenhouse-gas concentrations some future changes in the climate system already seem inevitable. Even if we drastically cut back our emissions and the atmospheric concentration of greenhouse gases stabilizes, the global average temperature is projected to continue to rise by a few tenths of a degree per century for a century or more, while sea level is projected to continue to rise for many centuries (IPCC, 2007a, p. 17). This is due to hysteresis in the climate system: it takes thousands of years for heat to be transported into the oceans and for a new equilibrium to be reached, and ice sheets are also likely to only respond slowly. But the projected climate changes also pose significant risks to societies and ecosystems in the nearer future (IPCC, 2007b). Already, recent regional changes in climate have been observed to affect ecosystems. Ecosystems are vulnerable to climate change and some are projected to become irreversibly damaged. Many human social systems are sensitive to climate change, with agricultural systems being especially vulnerable. Distributional aspects are important here, since societies with the least resources typically have the least capacity to adapt and are most vulnerable. To summarize: since some significant climate change is already projected to be nearly inevitable, adaptation to climate change will be a necessary element of climate policy. Furthermore, if we want to avoid taking the risk that the adverse impacts become even larger, we will need to mitigate the likely cause of climate change, that is, anthropogenic sources of greenhouse gases must be reduced and sinks must be enhanced.

Given that the climate change debate takes place in the highly polarized setting sketched above, how do science and its social and political context interact? The interaction goes in two ways. On the one hand, funding decisions are being made that have an impact on what science gets done and what science does not; and scientists bring in their own social and political values too in directing their research. The potential effects of the latter - oftentimes very subtle - interaction is addressed in the remaining sections of this paper.

on the other hand, scientists advise policy makers and politicians and inform society at large about their scientific findings. What roles can scientists play vis-à-vis policy makers and politicians? Pielke (2007) distinguishes between four idealized roles scientists may choose from: the roles of Pure Scientist (who seeks only the truth without considering the practical implications of his/her research), Science Arbiter (who seeks to focus on issues that can unequivocally be resolved by science), Issue Advocate (who seeks to advance particular interests using his/her expert status), or Honest Broker of Policy Alternatives. The latter role requires more explanation. For policy problems with large scientific uncertainties and 
high societal stakes, Hisschemöller et al. (2001) characterize the role of science in such a type of policy problem as that of 'problem recognizer'. The authority of scientists who take on this role can be assumed to reside in the scientists' ability to assess and communicate uncertainty and analyze the different values and perspectives on the problem: that is by being an Honest Broker. In practice, we find scientists taking on each of these four roles in the climate change debate. The media often feature Issue Advocates who are either pro or against climate policy measures, but who argue so on the basis of their reading of climate-simulation uncertainty (either read that uncertainty as being low or high) instead of being explicit about their underlying values. An example in the US context is the group of esteemed physicists who founded and led the influential George C. Marshall Institute and who joined the environmental backlash movement, using the argument that most of climate science is not good science (Lahsen, 2008).

In the case of climate change, climate scientists are quite certain about the fact that warming has occurred over the last five decades and judge it to be very likely that this warming largely results from anthropogenic causes (IPCC, 2007a, p. 10). Nevertheless there remain important scientific uncertainties which are of major importance for policy makers. Not only are there uncertainties concerning the specific causal processes of the observed climate change. In addition we are also confronted with uncertainties in future climate-change projections, uncertainties about the impacts of the predicted changes, uncertainties about the costs of these impacts, and, last but not least, uncertainties about the costs and benefits of different climate-change policies (adaptation and mitigation measures). These uncertainties need not be obstacles for political decision making on climate policy. This has been made explicit by the worlds' nations through including a formulation of the precautionary principle in the UNFCCC in 1992. Which actions are warranted, however, is understandably a matter of intense controversy. In this situation, the assessment of uncertainties and of the views held by the different actors involved in the policy debate, are crucial inputs for an informed political debate on the issue of climate change.

Just as people hold different views on what are acceptable risks, they also have different perspectives on uncertainties, including climate-simulation uncertainty. In the public debate on possible measures to curb $\mathrm{CO}_{2}$ emissions, critics of the proposed policy measures typically refer to uncertainties in climate simulation. They argue that there is no empirical evidence of the problem ('we don't see human-induced global warming happening yet') and that reliable prediction of climate far into the future (e.g., the year 2100) is not possible. As many of the critics currently admit that the earth's surface has warmed by about $0.5^{\circ} \mathrm{C}$ over the last 50 years, the alleged lack of evidence is basically a negative assessment of the quality of climate simulation. After all, it is only by combining the observations in an explanatory (= theoretical) model that one can attribute the observed changes to human influence rather than to natural fluctuations. From a philosophical point of view the critics certainly seem to have a case. Questioning the reliability of climate simulations is certainly legitimate. Hence the uncertainties involved in climate simulation have taken on a central role in the 'sound-science' debate (see e.g., Petersen, 2006b, Ch. 4) and to date a significant part of the political discussion on climate change has focused on the relationship between models and data (Edwards, 1999). Their perspective on the issue of climate change often leads lobbyists of the coal and oil industry to only take into account the lower range of the IPCC projections of future climate. They typically claim that climate sensitivity (the sensitivity of the global mean surface temperature to a doubling of $\mathrm{CO}_{2}$ ) is low.

At the other extreme, we find some lobbyists within the environmental movement who deem the upper range of the climate projections to be the most likely. Some environmentalists have criticized climate simulations for the fact that they would not be able to adequately model abrupt changes in the climate system (e.g. in the 1990s, Jeremy Leggett of Greenpeace; see Leggett, 1999). From this perspective, the climate system could well be too complicated to be modelled adequately. Ironically, the latter argument can also be used by the coal and oil lobby. However, they come to a different conclusion: we should not take the model outcomes seriously, because nothing is the matter. In contrast, the environmental movement argues for a precautionary approach, as is also adopted in the UNFCCC.

So we have scientists playing different roles towards policy making, as well as social and political values influencing scientific research. In this paper, I am particularly interested in the potential value-ladenness of climate simulation. It should be made clear at the outset, however, that there are different types of values which influence climate simulation practice. Kloprogge et al. (submitted) distinguish between two types of epistemic ${ }^{1}$ values (general epistemic values and discipline-bound epistemic values) and two types of nonepistemic values (socio-political values and practical values). The value diversity that is reflected in the different assumptions made in simulations can be regarded as a dimension of uncertainty (Petersen, 2006b, Ch. 3). Simulationists often have considerable freedom in making choices with respect to the conceptual and mathematical model; the model inputs; the technical model and the processing of output data and their interpretation. These choices are made either implicitly or explicitly. They have a subjective component and may be influenced by epistemic and non-epistemic values held by the simulationist. The choices thus have a potential to be valueladen. If the value-ladenness is indeed high for specific elements of the simulation and the results are significantly influenced by the value-laden choices made, then the simulation results

1 'Epistemic' means 'related to knowledge'. 
are also value-laden. In the remainder of this paper, the potential value-ladenness of climate simulation is explored, paying attention to the different values that may influence practice, including socio-political values.

\section{Hierarchy from simple to comprehensive climate models}

The complete set of families of climate models, which besides the well-known three-dimensional General Circulation Models (GCMs) also encompasses the much simpler zero-, one- and twodimensional models, is often referred to as the 'climate model hierarchy'. A consensus is presumed to exist within the climatescience community about the relative merits of the different climate model families - these model families typically play different functions. ${ }^{2}$ This 'consensus view' can be found in numerous publications. The overview presented here is based on Harvey et al. (1997). ${ }^{3}$ For each component of the climate system (atmosphere, oceans, terrestrial biosphere, glaciers and ice sheets, and land surface) a hierarchy of models can be identified. The main differences between these is in the number of spatial dimensions in the model (three, two, or one), the spatial and temporal resolution, the extent to which physical processes are explicitly represented (processes that are not explicitly represented have to be parameterized), the level of aggregation in the modelled system at which empirical parameterizations are involved, and the computational cost of running the model. ${ }^{4}$

According to the 'consensus view' both comprehensive and simple models have important roles to play in enhancing our understanding of the range of possible future climatic changes, their impacts, and interactive effects among the components of the climate system. Both pragmatic considerations involving computer resources and the level of detail appropriate to the coupling of the various components dictate the respective roles of comprehensive and simple climate models. The difference between comprehensive and simple climate models is presented by Harvey et al. (1997) in terms of the hierarchy of models introduced above. Typically, the behaviour of simple, 1-D climate models is easier to analyze, and sensitivity studies are easier to perform with simple models as compared to comprehensive models.
Both comprehensive and simple climate models contain empirical 'parameterizations', which are descriptions of processes not explicitly resolved in the models (e.g. convective cloud processes: these happen below the scale of a grid cell) but make use of parameters that are available at the grid scale (e.g. temperature and humidity). For comparison: all geophysical fluid dynamics models also make assumptions about the influence of processes smaller than the ones resolved in the models. One can either choose to neglect these processes, assuming they have no significant effect on the model results, or include the net effect of these processes through parameterizations. The difference between models at varying levels of comprehensiveness is that parameterizations are introduced at different levels of aggregation. For example, in a complex, 3-D climate model the smallest horizontal scale that can be resolved for the vertical transport of heat is a few hundred kilometres areally (smaller-scale transport needs to be parameterized), while in a simple, 1-D climate model all vertical transport of heat by atmospheric motion is parameterized. As an aside, it is noted by Harvey et al. (1997) that very highresolution models of clouds (large eddy simulation models) with a grid spacing of tens of meters and covering several tens of square kilometres have been developed. Note though that even such detailed models include parameterizations, e.g., parameterizations of cloud processes occurring at the micrometer scale. The ideal for some climate modellers is to extend these detailed models to the whole globe. However, this is presently not possible due to computing constraints.

A consequence of the fact that simple and comprehensive climate models have widely different resolutions is that in simple climate models in particular (for instance, 'upwellingdiffusion climate models') the climate sensitivity and other sub-system properties must be prescribed on the basis of results from comprehensive models or observations (if available). However, in comprehensive models, such properties are explicitly calculated from a combination of resolved processes and subgrid-scale parameterization in the models. ${ }^{5} \mathrm{~A}$ final, qualitative difference between simple and comprehensive models is related to predictability: One-dimensional simple climate models cannot simulate specific climatic 'surprises' like sudden major changes in the ocean circulation, while coupled Atmosphere-0cean GCMs (AOGCMs) can, although the

2 For an overview of different functions of climate simulation, see Müller \& Von Storch (2004) and Petersen (2006b, Ch. 5). For an essay book review on how models can be regarded as 'technological artefacts', see Petersen (2000).

3 The United Nations Framework Convention on Climate Change requested that the IPCC produce a technical paper (Harvey et al., 1997) on the characteristics, strengths, and weaknesses of simple climate models in relation to more complex ones. The main reason for this request was to document the simple climate models that were used for extensive sensitivity and scenario analysis in the Working Group I volume of the 1995 IPCC Second Assessment Report. In climate science, the term 'complex climate model' is often used instead of the term 'comprehensive climate model', which is preferred here, since simple climate models can also include non-linear feedbacks and thus model complexity, albeit simply.

4 Only a partially ordered hierarchy, based on the notion of 'complexity', can be defined since the complexity of the different model aspects (resolution, number of processes included, etc.) is not necessarily correlated: some models may include many processes at low resolution, or vice versa.

5 For completeness, it should be mentioned that in some other simple climate models (for instance, 'radiative-convective climate models') the climate sensitivity is simulated, albeit relying on a very crude parameterization. 
timing and the nature of such changes cannot currently be reliably ascertained. ${ }^{6}$

To sum up, depending on the question to be addressed there is usually a choice between different families of models that can be taken to the task. Each of these models has uncertainties associated with them. They are all reductions of real-world complexity. Some of these uncertainties are specific for a particular model (e.g., specific assumptions in the parameterization of clouds, see below), others for a particular family of models (e.g., the resolution needed to simulate internal climate variability) and again others are common for all models (e.g. radiative forcing from greenhouse gases).

\section{Methodological issues in climate modelling}

The structural features of climate simulation practice that are pivotal for the analysis in this paper are on the one hand the methodological rules that scientists aim at, or claim to follow in developing and evaluating simulations, and on the other hand the ultimate goals of scientific practice. Following Laudan (1984), I consider that there is a fundamental plurality of model assumptions, of methodologies for developing and evaluating models and of aims and goals of modelling. For the study of any given process by means of simulation, a multitude of simulation models can legitimately be used, even though the extent to which plurality is realized will vary depending on the context. Hence it is perfectly rational for simulationists to use different models, provided that they are willing to submit their models to each others' critical scrutiny.

During the production of the Second Assessment Report by the IPCC around the mid-1990s, it was observed, however, by Harvey et al. (1997) that the consensus of the climate modelling community was 'that detailed three-dimensional ... models of atmosphere and ocean dynamics, and correspondingly highly resolved models of the Earth's terrestrial and marine biota, are the long-term goals of Earth system science'. This statement reflects the climate-science community's choice to continue the approach of adding more complexity and spatial resolution to climate models. However, the consensus in the climate-science community was not as universal as is suggested by Harvey et al. (1997). Shackley et al. (1998), for instance, had identified within the scientific community two strong arguments against putting all the emphasis on this approach (both arguments are related to the fact that realization of a satisfactory comprehensive model seems distant):

1. Key parameterizations, for example, for clouds, are not fully theoretically based, and are hence often scale dependent and to some degree arbitrary. These parameterizations are crucial for climate model behaviour.
2. Models should be related to the spatial and temporal scale of the processes involved. It has not been validated that large-scale behaviour of climate can be represented by the combined effects of smaller-scale processes that are partly resolved and partly parameterized by complex climate models.

Although Shackley et al. (1998) did not aim to discredit the comprehensive climate-modelling approach, they did raise important questions concerning the methodology to be followed by climate scientists - questions which are still valid nowadays even though the specific examples may have changed.

Shackley et al.'s first argument makes clear that currently no climate model is of a theoretical high quality: ad hoc assumptions are systematically involved in deriving parameterizations. Simple and comprehensive climate models differ only in respect of the specific level of aggregation at which smaller-scale processes are parameterized. Thus both types of climate simulations suffer from the methodological problem of the arbitrariness of parameterizations, that is, their ad hoc character. An example of a major ad hoc, non-physical correction to comprehensive climate models that was widely applied in the 1990s is 'flux adjustment' - an ad hoc model fix that is introduced in coupling ocean and atmosphere general circulation models (resulting in coupled AOGCMs). This was necessary to be able to produce long enough computer simulation runs of an equilibrium climate. From a scientific perspective, flux adjustment is undesirable and the practice of flux adjustment is nowadays no longer widespread as in later versions of climate modelling software it is no longer needed. One of the reasons why in the year 2001 (as compared to the year 1996) the IPCC could state that 'confidence in the ability of models to project future climate has increased' (IPCC, 2001, p. 19), is related to the decreased dependence on flux adjustment:

"Some recent models produce satisfactory simulations of current climate without the need for non-physical adjustments of heat and water fluxes at the ocean-atmosphere interface used in earlier models." (IPCC 2001, p. 19)

Still, this 'increased confidence' does not negate the fact that most climate simulations that have been used in the first two IPCC reports (1990 and 1996) have made use of flux adjustment. This serves as a reminder that all numerical climate models include some ad hoc adjustments, even though the specifics of the adjustments may change over time. Current examples still include cloud parameterizations. ${ }^{7}$

Some scientists hold the ideal that ad hoc corrections should ultimately be removed. According to Randall and Wielicki (1997), for instance, parameterizations are often not strictly

\footnotetext{
6 Still, simple climate models also contain non-linearity's that may lead to the modelling of other types of surprises.

7 In surveys performed among climate scientists in 1996 and 2003, Bray and Von Storch (2007) both times found that atmospheric models were considered least able to deal with cloud processes.
} 
derived from and validated with observations, and they are typically not tested for many different conditions. Furthermore, the practice of tuning, that is, adjusting the model to observations without really understanding the physical processes that are being modelled, is considered problematic by them. They argue that a parameterization should be left unchanged when the model that includes the parameterization is tested as a whole. Accordingly, one should refrain from tuning the parameterizations interactively in order to have the outcomes of the model match all available observations, since this 'bad' practice cannot give one confidence in the predictive power of the model. Tuning the empirical components is allowable only if a process is very important and poorly understood. One must subsequently strive, through continuing research, to arrive at a good understanding of how to parameterize the process. If one has succeeded in that, the tuning of the model can subsequently be removed. The quality of a parameterization depends both on its calibration with observations and on its theoretical underpinning. According to purist modellers, in particular, a parameterization can be considered more reliable if its theoretical underpinning is strong.

There are two methodological problems related to Randall and Wielicki's outright rejection of tuning. Firstly, their methodological proposal makes the assumption that in principle all tuning should ultimately be eliminated. However, this is not at all self evident. Of course, the elimination of some of the adjustable parameters from the current models can lead to an improvement of these models. There is no guarantee though that by progressively eliminating all ad hoc corrections (even if that were possible) numerical climate models will ever acquire good predictive capacities. The Dutch meteorologist Henk Tennekes doubts whether the whole project is even possible. He observes: 'In practice a computer model always contains all sort of tricks and empirical rules, no matter how many refinements are added. The empiricism (empirical content) contained in a computer model cannot be adjusted in advance; it is tuned by repeatedly checking the performance of the model against observations, until the model finally functions in a reliable way. (Since) the climate is a one-time experiment ..., the predictions of climatic models are always overtaken by the facts, regardless of how reliable the models are' (Tennekes, 1994, pp. 78-79). In other words, the climate of the future is fundamentally unpredictable.

Secondly, the testing of climate models is not a straightforward matter. Verification of a model is logically impossible (Popper, 1959). Even if we use the growing observational record to test climate models, the question of their reliability still has to be dealt with when climate models are used to predict future climate change. The approach the scientific community has taken is to link the predictive capability of numerical climate models to their performance in reproducing the historical record (both the geological record and the past period of about 150 years for which we have a global record of real-time temperature observations). If we apply Popper's (1959) philosophy about theories to models, we can claim that if the models do not fail in this regard, they should be considered to be 'corroborated' (corroboration is a matter of degree and depends on the severity of the tests to which the models have been put). The impossibility of establishing the absolute truth of a theory has led Popper to insist on falsifiability as the hallmark of the scientific method. Following the same line of reasoning, Randall and Wielicki (1997) - who take a model to be an embodiment of a theory, thus providing a scientific basis for predictive modelling - argue that model predictions can be proven wrong or falsified by comparison with measurements that the model was supposed to predict, and that one should strive for such falsification.

Randall and Wielicki (1997) consider falsification of the whole model (including both the 'principal hypothesis' and 'auxiliary hypothesis') possible. In Popperian fashion, this methodology takes climate models to be corroborated by each unsuccessful attempt to falsify the model. The method proposed by Randall and Wielicki (1997) is more difficult to apply for comprehensive than for simple models. One reason being that current comprehensive models involve tuning of many of the parameterizations within the model. If the model agrees with the observations against which it is tested, this could be the result of 'compensating errors' (namely, the model has been tuned as a whole). Consequently if the model does not agree with the observations, one does not know which adjustable parameters (or even complete parameterizations) are wrong. The testing of models therefore has to be carefully framed. This is the key message in the discussion by Randall and Wielicki (1997) on the falsification of models.

Shackley et al.'s second argument against focusing too much on comprehensive climate models raises questions related to the complexity of climate (see also Rind, 1999). It may be the case that the processes that occur across the wide range of scales modelled by comprehensive climate models can, in fact, be addressed separately for different sub-ranges of scales. This ultimately depends on the importance of the smallest-scale non-linearities for the large spatial and temporal scales that are considered in climate change studies. In view of the policy context of climate simulation a crucial question is, what are the spatial and temporal scales needed to accurately simulate globally averaged climate change at a 100-year time-scale? The current answer is that we don't know - we should recognize our ignorance about this issue. We do not know the degree to which feedbacks within the climate system are influenced by non-linearities in the climate system and their effect on future patterns of variability (Rind, 1999). In other words, we do not know whether or not a model of the climate system can be constructed as a hierarchy of dynamically uncoupled models (ordered by characteristic time-scales) across a broad range of temporal scales (cf. Werner, 1999).

The IPCC technical paper (Harvey et al., 1997) was an attempt by the climate-science community to address the 
methodological questions of complexity through an assessment forum (although this was not the first aim of that paper). The fact that the IPCC technical paper got no further than pointing out that in the scientific climate-assessment practice pragmatic use is made of both comprehensive and simple climate models, may lead to the conclusion that no universally agreed-upon methodology for climate modelling exists among climatesimulation practitioners (cf. Shackley et al., 1998). The methodological analysis by Harvey et al. (1997) claims merely that climate modelling is an 'art' and that there is 'no methodological crank to turn'. This is a position that I disagree with, since even though a plurality of methodologies exists, it is possible and desirable to discuss and compare the various methodologies. ${ }^{8} \mathrm{~A}$ nice example of such a methodological discussion within the climate modelling literature is a paper by Isaac Held (2005) in the Bulletin of American Meteorological Society. After noting that the importance of a climate modelling hierarchy has often been emphasized, he continues:

"But, despite notable exceptions in a few subfields, climate theory has not, in my opinion, been very successful at hierarchy construction." (Held, 2005, p. 1609)

Held questions whether the comprehensive climate models really lead to improved understanding. He argues that we need simple models that capture the essential dynamics of the phenomenon being investigated, in order to check whether we really understand what is happening in the comprehensive models. According to Held, 'the health of climate theory/ modelling in the coming decades is threatened by a growing gap between high-end simulations and idealized theoretical work. In order to fill this gap, research with a hierarchy of models is needed' (Held, 2005, p. 1614).

From the preceding discussion on the relative merits of comprehensive and simple climate models, we can infer that the plurality at the methodological level is correlated with a plurality at the level of aims and goals of simulation: different climate scientists may entertain different goals of simulation in their climate-simulation practice. Can we discern such a correlation between methods and aims amongst climatesimulation laboratories? The following discussion on the social and political context of climate-simulation practice addresses that question.

\section{The value-ladenness of climate-simulation practice}

The social and political context in which climate simulations are developed, evaluated and applied has a significant influence on climate-simulation practice, as has been shown in the sociological work of Simon Shackley and co-workers, among other authors. Here I will first summarize what Shackley et al. found (Shackley et al., 1999; Shackley, 2001). They identified different styles of doing climate simulation within the research community, with the different styles embodying different standards by which to evaluate simulation models and their results.

Shackley et al. (1999) make a distinction between two styles, a 'pragmatist' one and a 'purist' one. Taking the example of 'flux adjustment', the authors show that while pragmatists consider flux adjustment to be sufficiently innocent to represent the ocean-atmosphere coupling and still arrive at meaningful results, purists 'apply seemingly more rigorous, yet still private and informal standards of model adequacy' (Shackley et al., 1999, p. 428). These authors are wary of flux adjustments as 'potentially covering-up model errors, influencing the model's variability, and leading to complacency in model improvements' (Shackley et al., 1999, p. 445). By using flux adjustment the climate modellers were able both to present results to policy makers and to point out the need for further model development. Pragmatic and purist modellers do not usually make their tacit criteria explicit in their publications, nor are the differences of opinion concerning the use of climate simulation results for policy development mentioned in the IPCC reports. Why pragmatists find the application of flux adjustment acceptable for the production of policy-relevant climate runs remains hidden from view, since 'perceptions of policy needs are built seamlessly into scientific interactions' (Shackley et al., 1999, p. 435).

A range of factors plays a role in determining both the existence of the two cultures and their membership. Shackley et al. (1999) observe that:

"pragmatist and purist cultures emerge from the interplay of a heterogeneous range of factors including: organizational mission, individual and collective research trajectories (including past work experience and identification of future priorities and ambitions), funding patterns, involvement in providing climate-impacts scientists with scenarios, the role of hierarchical management and/or charisma of leaders of research groups, and different epistemic styles." (Shackley et al., 1999, p. 445)

These heterogeneous factors together constitute the social and political context of climate simulation. The distinction made between the two cultures sheds light on the fundamental assumptions of different modelling approaches. Many of the pragmatists do believe that it is correct to assume the impact of flux adjustment to be small with respect to the overall results of their models. However, they have not been able to convince the purists, who believe the assumption might be incorrect and inappropriate for use. Here we encounter an uncertainty of the 'recognized ignorance' type. In my view, at

8 This is not to say that Harvey et al. (1997) do not recognize the importance of simple climate models. As in other climate modelling 'primers' (e.g. McGuffie and Henderson-Sellers, 1997), the value of simple climate models is acknowledged. 
the time this controversy raged we had to remain open as to the question which of the schools would in the future be judged to have been right. ${ }^{9}$ Not only epistemic considerations but also social considerations play a role in the choices that individual modellers make. The choice to employ flux adjustment in climate simulations intended to support policy advice was in my view clearly value-laden.

In an investigation of the use of comprehensive models in climate-change simulations, Shackley (2001) compared two American climate-modelling groups: the Geophysical Fluid Dynamics Laboratory (GFDL, Princeton, NJ) and the National Center for Atmospheric Research (NCAR, Boulder, CO). The comparison was with each other and with the UK climatemodelling group, the Hadley Centre for Climate Predication and Research (Bracknell, England ${ }^{10}$ ). In that publication, Shackley identified the following three 'epistemic lifestyles' in the comprehensive climate-modelling community as a whole, the first two of which correspond to respectively the pragmatist and purist styles discussed earlier:

1. Climate seers. 'Those conducting model-based experiments to understand and explore the climate system, with particular emphasis on its sensitivity to changing variables and processes, especially increasing greenhouse gas concentrations' (Shackley, 2001, p. 115). This style is similar to the pragmatist style identified in Shackley et al. (1999) and is dominant within GFDL. The specific function of climate simulation within this style - aside from being a substitute for experiments - is that of a heuristic tool to develop hypotheses about climate change.

2. Climate-model constructors. "Those developing models that aim to capture the full complexity of the climate system, and that can then be used for various applications' (Shackley, 2001, p. 115). This style compares with the purist style and is dominant within NCAR. Within this style - again in addition to being a substitute for experiments - simulation functions as a technique to investigate the detailed dynamics of the climate system.

3. Hybrid climate-modelling/policy style. "The policy-influenced objectives and priorities of the research organization, as defined by its leadership, take precedence over other individual or organizational motivations and styles' (Shackley, 2001, p. 128). The Hadley Centre is an example. One of the objectives of the Centre is to perform climate simulations that can be used as input to the assessment processes by the IPCC. ${ }^{11}$ Both climate seers and climatemodel constructors are involved in the Centre, but none of these styles dominates due to the hierarchical style of management which enables the Centre to be both policydriven and of a high scientific quality.
Shackley (2001, p. 129) observes that a range of factors influences which epistemic lifestyle is adopted in a climatemodelling centre. Factors that matter include disciplinary/ research experience background, organizational location and objectives, main funders, main user and customers, the role of academic collaborators and users of models, the role of policy makers in negotiations over research priorities and directions, the role of organizational culture, the opportunities to treat the climate model as a 'boundary object' (e.g. between climate and numerical weather prediction research), and the role of different national cultures of research.

One of the influences on the choice of epistemic lifestyle is constituted by the political views of the climate scientists themselves on the climate-change problem. Bray and Von Storch $(1999 ; 2008)$ found systematic differences in climate scientists' political views at a national scale. Based on international surveys among climate scientists they concluded that North American climate scientists perceived the need for societal and political responses to be less urgent than their German counterparts. These differences also correlate with different assessments of the quality of climate simulations. Bray and Von Storch (1999) report that even though almost all climate scientists agree that the quality of climate-simulation models is limited, the U.S. scientists were less convinced of the quality of the models than their German counterparts.

Since the 'climate seers' prefer relatively simple models and the 'climate-model constructors' prefer relatively comprehensive models, the plurality of epistemic lifestyles within and among climate-simulation laboratories thus leads to different assessments of the relationship between simple and comprehensive climate models. We can conclude therefore that there is no universally agreed-upon methodology for climate simulation and that different groups of climate scientists entertain different goals of climate simulation. When we assess the uncertainties in climate simulation, we should therefore pay attention to the potential value-ladenness (including sociopolitical values) of the choices made by individual modellers or modelling groups.

\section{Evaluating the plurality of climate-simulation models}

A wide variety of methodological approaches exists for building and evaluating simulation models. In this paper the case for pursuing pluralism in climate modelling is argued. Parker (2006) also observes that although climate models incorporate mutually incompatible assumptions about the climate system (e.g., different physical assumptions in cloud parameterizations), they are used together as complementary resources for

9 As was said in the previous section, it now seems that the pragmatists had been correct in this specific case of flux adjustment.

10 The Hadley Centre and the Met0ffice both moved to Exeter in 2003.

11 The IPCC assessments give most weight to results that have been published in internationally peer-reviewed journals. Hence the Hadley Centre, like other climatemodelling groups, publishes its results in journals such as Nature. 
investigating future climate change. Indeed, climate modellers are well aware of the limitations of their models and therefore within the context of the IPCC 'ensembles' of models are used to assess what is happening to the climate system.

How is this to be understood philosophically? Parker observes both an 'ontic competitive pluralism'12 and a 'pragmatic integrative pluralism' in climate-simulation practice. Ontic competitive pluralism exists when two models make conflicting claims about the same part of the world that they are intended to model. Typically, these models are then viewed as 'competitors'. This contrasts with 'ontic compatible pluralism', which exists when there are two or more representations of the world that can both be true at the same time. From the preceding analysis I conclude that the different types of climate models make mutually conflicting claims about how the climate system behaves. Climate scientists such as Randall and Wielicki would like to select from among the comprehensive climate models the one which actually incorporates the most realistic assumptions about the physical processes that influence climate. Parker (2006) points out, however, that for a variety of reasons, scientists have simply been unable to identify such a model.

How can climate models be possibly combined meaningfully in 'multi-model ensembles' if they are incompatible? According to Parker, the pluralism in climate modelling is also 'integrative', in the sense that different climate models are used together to investigate the scientific uncertainty about the climate system. Parker concludes that climate models are thus compatible not at the level of ontology but at the level of practical application. Hence her term 'pragmatic integrative pluralism', which reflects the awareness of the climate scientists that probably none of their models are correct. Thus, two types of pluralism - ontic competitive pluralism and pragmatic integrative pluralism co-exist in climate-simulation practice.

However, some climate scientists and policy makers regard the plurality of epistemic lifestyles in climate simulation as a problem. A typical example of this attitude can be found in the report "The Capacity of U.S. Climate Modelling to Support Climate Change Assessment Activities' by the Climate Research Committee of the U.S. National Research Council (1998). The Committee argues that the hybrid climate-modelling/policy style of European climate modelling centres should be copied in the United States, since 'the United States lags behind other countries in its ability to model long-term climate change' (National Research Council, 1998, p. 5). While 'the U.S. climate modelling research community is a world leader in intermediate and smaller climate modelling efforts' (National Research Council, 1998 , p. 1), comparatively little money has been invested in developing and running high-end, comprehensive climate models.
But from a methodological point of view, the diversity in climate modelling efforts, partly reflecting differences in the social organization of research, must be valued positively, given the large uncertainties about the behaviour of the climate system. There are only a few centres where the most comprehensive climate models are developed and no 'fully satisfactory systematic bottom-up approach' (Held, 2005, p. 1611) for developing these comprehensive models is available. In fact,

"model builders put forward various ideas based on their wisdom and experience, as well as their idiosyncratic interests and prejudices. Model improvements are often the result of serendipity rather than systematic analysis. Generated by these informed random walks, and being evaluated with different criteria, the comprehensive climate models developed by various groups around the world evolve along distinct paths." (Held, 2005, p. 1611)

By building models of 'intermediate complexity' (Claussen et al., 2002) that are sufficiently complex to allow for the simulation of processes of interest but are easier to understand than the most complex models, it is possible to gain a better understanding of which parameterizations determine the main uncertainties of the comprehensive models. This makes more informed choices in model development possible.

To conclude, I have shown that climate models of varying levels of concreteness and of varying basic assumptions exist and are valued differently by different groups of climate scientists. The social and political context in which the different groups of scientists operate plays a role in how their results are valued. In order to prevent one particular group of models from dominating the field, the climate-modelling community should acknowledge the vital and necessary role of plurality in the practice of climate science and should stimulate reflection among practitioners. This could help to improve the communication of uncertainties in climate simulation to policy makers and politicians (cf. Wardekker et al., 2008).

\section{Acknowledgements}

This paper is largely the fruit of dissertation research performed by the author at the Faculty of Philosophy, Vrije Universiteit Amsterdam. Hans Radder and Peter Kirschenmann are thanked for guiding him through this project. The author furthermore thanks one anonymous reviewer for his helpful comments. 


\section{References}

Bray, D. \& Von Storch, H., 1999. Climate science: an empirical example of postnormal science. Bulletin of the American Meteorological Society 80: 440-455.

Bray, D. \& Von Storch, H., 2007. Climate Scientists' Perceptions of Climate Change Science, GKSS Report 2007/11. Institute for Coastal Research GKSS Forschungszentrum (Geesthacht) Germany: 124 pp. Available at: http://dvsun3.gkss.de/BERICHTE/GKSS_Berichte_2007/GKSS_2007_11.pdf.

Claussen, M., Mysak, L.A., Weaver, A.J., Crucifix, M., Fichefet, T., Loutre, M.-F., Weber, S.L., Alcamo, J., Alexeev, V.A., Berger, A., Calov, R., Ganopolski, A., Goosse, H., Lohmann, G., Lunkeit, F., Mokhov, I.I., Petoukhov, V., Stone, P. \& Wang, Z., 2002. Earth system models of intermediate complexity: closing the gap in the spectrum of climate system models. Climate Dynamics 18: 579-586.

Edwards, P.N., 1999. Global climate science, uncertainty and politics: dataladen models, model-filtered data. Science as Culture 8: 437-472.

Funtowicz, S.O. \& Ravetz, J.R., 1993. Science for the post-normal age. Futures 25: 739-755.

Harvey, L.D.D. \& co-authors, 1997. An Introduction to Simple Climate Models Used in the IPCC Second Assessment Report, IPCC Technical Paper 2. Intergovernmental Panel on Climate Change (Geneva): 50 pp. Available at: http://www.ipcc.ch/pdf/technical-papers/paper-II-en.pdf.

Held, I.M., 2005. The gap between simulation and understanding in climate modeling. Bulletin of the American Meteorological Society 86: 1609-1614.

Hisschemöller, M., Hoppe, R., Groenewegen, P. \& Midden, C.J.H., 2001. Knowledge use and political choice in Dutch environmental policy: a problemstructuring perspective on real life experiments in extended peer review. In: Hisschemöller, M., Hoppe, R., Dunn, W.N. \& Ravetz, J.R. (eds): Knowledge, Power, and Participation in Environmental Policy Analysis. Transaction Publishers (New Brunswick) USA: 437-470.

IPCC, 2001. Climate Change 2001: The Scientific Basis. Contribution of Working Group I to the Third Assessment Report of the Intergovernmental Panel on Climate Change (Houghton, J.T., Ding, Y., Griggs, D.J., Noguer, M., van der Linden, P.J., Dai, X., Maskell, K. \& Johnson, C.A. (eds)). Cambridge University Press (Cambridge) United Kingdom \& (New York) NY, USA: 881 pp.

IPCC, 2007a. Climate Change 2007: The Physical Science Basis. Contribution of Working Group I to the Fourth Assessment Report of the Intergovernmental Panel on Climate Change (Solomon, S., Qin, D., Manning, M., Chen, Z., Marquis, M., Averyt, K.B., Tignor, M. \& Miller, H.L. (eds)). Cambridge University Press (Cambridge) United Kingdom \& (New York) NY, USA: 996 pp.

IPCC, 2007b. Climate Change 2007: Impacts, Adaptation and Vulnerability. Contribution of Working Group II to the Fourth Assessment Report of the Intergovernmental Panel on Climate Change (Parry, M.L, Canziani, O.F., Palutikof, J.P., Van der Linden, P.J. \& Hanson, C.E. (eds)). Cambridge University Press (Cambridge) United Kingdom \& (New York) NY, USA: 976pp.

IPCC, 2007c. Climate Change 2007: Mitigation. Contribution of Working Group III to the Fourth Assessment Report of the Intergovernmental Panel on Climate Change (Metz, B., Davidson, 0.R., Bosch, P.R., Dave, R. \& Meyer, L.A. (eds)). Cambridge University Press (Cambridge) United Kingdom \& (New York) NY, USA: $851 \mathrm{pp}$.

Kloprogge, P., Van der Sluijs, J.P. \& Petersen, A.C., submitted. A method for the analysis of assumptions in model-based environmental assessments.
Lahsen, M., 2008. Experiences of modernity in the greenhouse: a cultural analysis of a physicist 'trio' supporting the backlash against global warming. Global Environmental Change 18: 204-219.

Laudan, L., 1984. Science and Values: The Aims of Science and Their Role in Scientific Debate. University of California Press (Berkeley) CA, USA: 149 pp.

Leggett, J., 1999. The Carbon War: Global Warming at the End of the Oil Era. Penguin Books (London) UK: 342 pp.

McGuffie, K. \& Henderson-Sellers, A., 1997. A Climate Modelling Primer, 2nd edition. John Wiley \& Sons (New York): 268 pp.

Müller, P. \& Von Storch, H., 2004. Computer Modelling in Atmospheric and 0ceanic Sciences: Building Knowledge. Springer (Berlin, Heidelberg \& New York): $304 \mathrm{pp}$.

National Research Council, 1998. The Capacity of U.S. Climate Modeling to Support Climate Change Assessment Activities. National Academy Press (Washington DC) USA: 78 pp.

Parker, W.S., 2006. Understanding pluralism in climate modelling. Foundations of Science 11: 349-368.

Petersen, A.C., 2000. Models as technological artefacts. Social Studies of Science 30: 793-799.

Petersen, A.C., 2006a. Simulation uncertainty and the challenge of postnormal science. In: Lenhard, J., Küppers, G. \& Shinn, T. (eds): Simulation: Pragmatic Constructions of Reality - Sociology of the Sciences, vol. 25. Springer (Dordrecht) the Netherlands: 173-185.

Petersen, A.C., 2006b. Simulating Nature: A Philosophical Study of ComputerSimulation Uncertainties and Their Role in Climate Science and Policy Advice. Het Spinhuis Publishers (Apeldoorn) the Netherlands \& (Antwerp) Belgium: 210 pp. Available at: http://hdl.handle.net/1871/11385.

Pielke, R.A., 2007. The Honest Broker: Making Sense of Science in Policy and Politics. Cambridge University Press (Cambridge) UK: 188 pp.

Popper, K.R., (1934) 1959. The Logic of Scientific Discovery. Routledge (London) UK: $480 \mathrm{pp}$.

Randall, D.A. \& Wielicki, B.A., 1997. Measurements, models, and hypotheses in the atmospheric sciences. Bulletin of the American Meteorological Society 78: 399-406.

Rind, D., 1999. Complexity and climate. Science 284: 105-107.

Shackley, S., 2001. Epistemic lifestyles in climate change modeling. In: Miller, C.A. \& Edwards, P.N. (eds): Changing the Atmosphere: Expert Knowledge and Environmental Governance. MIT Press (Cambridge) Ma, USA: 107-133.

Shackley, S., Young, P., Parkinson, S. \& Wynne, B., 1998. Uncertainty, complexity and concepts of good science in climate change modeling: are GCMs the best tools? Climatic Change 38: 155-201.

Shackley, S., Risbey, J., Stone, P. \& Wynne, B., 1999. Adjusting to policy expectations in climate change modeling: an interdisciplinary study of flux adjustments in coupled atmosphere-ocean general circulation models. Climatic Change 43: 413-454.

Swart, R., Bernstein, L., Ha-Duong, M. \& Petersen, A., 2008. Agreeing to disagree: uncertainty management in assessing climate change, impacts and responses by the IPCC. Climatic Change, in press, doi:10.1007/s10584-0089444-7: 29 pp.

Tennekes, H., 1994. The limits of science. In: Zweers, W. \& Boersema, J.J. (eds.): Ecology, Technology and Culture. The White Horse Press (Cambridge) UK: 72-88. 
Van Asselt, H., Berghuis, J., Biermann, F., Cornelisse, C., Haug, C., Gupta, J. \& Massey, E., 2008. Exploring the Socio-Political Dimensions of Climate Change Mitigation, IVM Report W-08/18. Institute for Environmental Studies, Vrije Universiteit (Amsterdam): 104 pp. Available at: http://www.ivm.vu.nl/ images_upload/4BC45814-D58C-1894-9C415605D27FB5B1.pdf.

Van der Sluijs, J.P., Petersen, A.C., Janssen, P.H.M., Risbey, J.S. \& Ravetz,

J.R., 2008. Exploring the quality of evidence for complex and contested policy decisions. Environmental Research Letters 3: 024008: 9 pp.

Wardekker, J.A., Van der Sluijs, J.P., Janssen, P.H.M., Kloprogge, P. \&

Petersen, A.C., 2008. Uncertainty communication in environmental assessments: views from the Dutch science-policy interface. Environmental Science \& Policy 11: 627-641.

Werner, B.T., 1999. Complexity in natural landform patterns. Science 284: 102-104. 